\title{
A Multi-Floor Indoor Pedestrian Localization Method Using Landmarks Detection for Different Holding Styles
}

\author{
Khanh Nguyen-Huu ${ }^{1}{ }^{1}$ and Seon-Woo Lee $\mathbb{D D}^{1,2}$ \\ ${ }^{1}$ Research Institute of Information and Electronic Engineering, Hallym University, Chuncheon, Republic of Korea \\ ${ }^{2}$ School of Software, Hallym University, Chuncheon, Republic of Korea \\ Correspondence should be addressed to Seon-Woo Lee; senu@hallym.ac.kr
}

Received 17 November 2020; Revised 23 January 2021; Accepted 13 February 2021; Published 1 March 2021

Academic Editor: Jose F. Monserrat

Copyright ( 2021 Khanh Nguyen-Huu and Seon-Woo Lee. This is an open access article distributed under the Creative Commons Attribution License, which permits unrestricted use, distribution, and reproduction in any medium, provided the original work is properly cited.

\begin{abstract}
The pedestrian dead reckoning (PDR) technique is widely used due to its ease of implementation on portable devices such as smartphones. However, the position error that accumulates over time is the main drawback of this technology. In this paper, we propose a fusion method combining a PDR technique and the landmark recognition methods for multi-floor indoor environments using a smartphone in different holding styles. The proposed method attempts to calibrate the position of a pedestrian by detecting whether the pedestrian passes by specific locations called landmarks. Three kinds of landmarks are defined, which are the $\mathrm{WiFi}$, the turning, and the stairs landmarks, and the detection methods for each landmark are proposed. Besides, an adaptive floor detection method using a barometer and a WiFi fingerprinting technique is suggested for tracking a pedestrian in a multifloor building. The developed system can track the pedestrian holding a smartphone in four styles. The results of the experiment conducted by three subjects changing the holding style in a three-floor building show the superior performance of the proposed method. It reduces the error rate of positioning results to less than $57.51 \%$ compared with the improved PDR alone system.
\end{abstract}

\section{Introduction}

In recent years, the attention and demand for indoor location-based services have been increasing due to its wide range of applications such as emergency rescue and surveillance in buildings. The global navigation satellite system such as the global positioning system (GPS) typically works well for outdoor environments, but in the case of indoor ones, it fails to provide good results. Two types of indoor localization systems that attract a lot of attention are the radio-based methods and the dead reckoning technique for pedestrian localization, known as pedestrian dead reckoning (PDR) [1]. The former can solve the localization problem in two ways: the triangulation and the fingerprint. The major limitations of this type of technique are time-consuming and expensive initial installation costs. The latter type, which uses inertial sensors to estimate the displacement of the user based on the user's previous position, is also a promising technique due to the absence of any infrastructure. The PDR only requires lightweight and inexpensive sensors which can be found easily on many portable devices such as smartphones, smartwatches, and tablets. Its major drawback, however, is the accumulation of errors over time or distance traveled. To handle this problem, some researchers have applied different kinds of filters such as the Kalman filter [2-5] or the particle filter [6-9]. Some researchers used special locations such as stairs or corners, to calibrate the position of the user. The WiFi fingerprinting technique is one example that can help the PDR system [10-14]. Activitybased map matching $[15,16]$ focuses on the detection of different activities or events such as taking the escalator or elevator and going up or downstairs. Magnetic-based map matching methods $[7,17,18]$ have also been suggested to improve the performance of the conventional PDR system. These works can be considered as fusing schemes of a PDR technique and attempts to remove the cumulative errors.

We propose a fusion method combining a PDR technique and the landmark recognition methods for multi-floor 
indoor environments using a smartphone in four holding styles. The proposed method attempts to calibrate the position of a pedestrian by detecting whether he or she passes by specific locations called landmarks. Three kinds of landmarks are defined, which are the $\mathrm{WiFi}$, the turning, and the stairs landmarks, and the detection methods for each landmark are proposed. In addition, a simple but robust floor detection method using a barometer and a WiFi fingerprinting technique is suggested. Due to this floor detection capability, the developed system can track a pedestrian walking in a multi-floor building. The main contributions of this work are listed as follows:

(i) A hybrid pedestrian localization method is proposed. The method tries to track the position of a pedestrian holding a smartphone in four styles based on the outputs of an improved PDR and landmark recognition.

(ii) Three kinds of landmarks as the compensators are introduced to correct the errors, and methods to detect the landmarks are proposed.

(iii) A robust method to estimate the current floor level and the detection of interfloor changes is suggested. The proposed approach uses the advantages of the WiFi fingerprinting technique and a barometer.

(iv) The proposed method is implemented on the Android platform to evaluate the performance in realtime. Various experiments were conducted to verify the usefulness of the proposed method. In particular, the performance according to the four holding styles was analyzed. Based on the experimental results, a comparative analysis was performed with other related approaches.

\section{Related Works}

Harle [1] made a survey for developments in the dead reckoning for walking users and hybrid systems using PDR techniques. He reviewed several techniques for detecting the walking step, estimating the step length, and calculating the heading of the user for two types of localization systems, the inertial navigation systems (INSs), and the step and heading systems (SHSs). He concluded that the traditional PDR approach can work well in a short-to-medium term tracking, but it needs the help of other absolute localization systems.

To calibrate a PDR system, radio signal-based systems have been developed. Zhuang et al. [3] provided an integrated system that consists of PDR, INS, and WiFi. The proposed system used the extended Kalman filter to combine the position information from PDR/INS/WiFi components. The average accuracy of the system is $4.5 \mathrm{~m}$. The same approach was developed by Deng et al. [11] which also used the extended Kalman filter to fuse different information from PDR, WiFi, and some special locations (i.e., elevators, escalators, and doors) in order to reduce the error. The average positioning error of this method was only $1.22 \mathrm{~m}$. With the support of the Bluetooth beacons, a $2 \mathrm{~m}$ precision PDR system was demonstrated by Li et al. [19]. Using the
RFID, Seco and Jimenez [20] applied the particle filter to fuse the information of PDR and active RFID nodes, resulting in an under- $2 \mathrm{~m}$ error. The suggested infrastructure-assisted PDR approaches could reduce considerably the amount of time and effort of making the preliminary offline map construction, compared with the system without PDR capability. Wang et al. [14] suggested a fusion method called landmark-aided PDR (LaP) to correct the cumulative error from the PDR by detecting landmarks. They defined WiFi signal-based landmarks and the turning activity landmark. Despite its simplicity, the method performed better than other complex methods. The basic idea of this fusion method is the same as ours, but our proposed method is different in that it defines and recognizes more landmarks, responds to various holding styles, and applies to multi-floor environments.

Activity-based map matching methods also can calibrate the position error by detecting user's activities and matching their activities to corresponding specific positions. Elhoushi et al. [16] attempted to classify the activities of a pedestrian between walking on a floor plan or stairs and taking an escalator or elevator. Based on each detected activity, they treated their PDR system in different ways. A better positional accuracy was shown at the endpoint with $2.2 \mathrm{~m}$. Park et al. [21] suggested a calibration method of PDR using the hidden Markov model (HMM) and a detection method of corners as landmarks using a magnetometer and an accelerometer. Zhou et al. [15] have proposed an interesting hybrid PDR system called the activity sequence-based indoor pedestrian localization system, which uses HMM and the detection of special activities such as turning at a corner, taking the elevator or escalator, and walking stairs. Using HMM and the introduction of a network of nodes (equivalent to landmarks), they could release the assumption that the system knows the initial position in prior.

The PDR technique can only estimate the position on a plane. To make it possible to multi-story buildings, a localization system could recognize the floor level. It is often thought that the conventional WiFi fingerprinting method solves this problem easily; however, it is not due to the complexity or the irregularity of the structure of a building. Zhao et al. [22] pointed out that at well-partitioned areas (i.e., thick ceilings), the difference of the received signal strength indicator (RSSI) values between two adjacent floors is large so it could be used to estimate the floor level. However, at hollow areas (i.e., stairways), the signal variation and the small signal propagation attenuation between adjacent floors will make it impossible to identify the floor level well. Elbakly et al. [23] attempted to estimate the floor level using a neural network. The proposed method achieved an accuracy of over $90 \%$.

Nowadays barometers are widely integrated into many smartphones, so they can be used to determine the floor level. The major problems of using barometers are device heterogeneity and variations due to the changes in temperature and wind. Shen et al. [24] and Zhao et al. [22] suggested hybrid floor identification methods that utilize the advantages of WiFi and barometers. These systems can achieve accuracies of floor detection of over $95 \%$. This is 
different from our system, which uses a simple but robust adaptive lookup table approach. Our floor detection method uses the Wifi fingerprinting method and a barometer like this approach; however, unlike previous studies, it uses the idea of applying two methods separately depending on the stationary and moving state. The proposed method uses an adaptive lookup table to provide a simple but robust performance.

\section{The Proposed Method}

3.1. Overview. A block diagram of the proposed system is shown in Figure 1, which is composed of three main components: the improved PDR, landmark recognition, and floor detection. A position coordinator is also included in the diagram. The system operates in two modes, the stopping mode and the walking mode. When the user does not walk for more than 10 minutes, the system is set to the stopping mode. If a new walking step is detected, then it is set to the walking mode.

The improved PDR component (i-PDR) means a conventional PDR method with an improved capability of step detection, step length estimation, and measuring the heading of walking using the recognition of holding styles, which was reported in our previous works $[25,26]$.

One major advantage of our proposed method is freeing the user from a fixed smartphone holding style, by recognizing four styles: holding the phone in one's hand in front of the body (HA), holding the phone parallel to the ears like calling (CA), swinging the phone during walking (SW), and putting the phone into the pants' pocket at the front (PO). The holding styles are classified using a decision tree method (J48 classifier) with 30 features. The accuracy of the classification is over $99 \%$. Based on the detected holding style, our approach then uses different methods to detect a new walking step and to estimate the step length. It is well known that a remaining critical issue in PDR is the estimation of the walking direction [27]. The proposed heading estimation method uses the quantization technique with hysteresis property that the measured heading is mapped to one among the eight directions. The central value of each direction was chosen according to the major directions of the corridors in given buildings. This idea is similar to the proposed approach using dominant directions by Ju et al. [28]. The PDR component outputs a $2 \mathrm{D}$ position as $(x(t), y(t))$. The sampling frequency of the reading sensors is $30 \mathrm{~Hz}$. The moment of detecting a new step is considered as another kind of major sampling time.

The landmark recognition component detects whether the user walks on the landmarks. Three kinds of landmarks are defined. Firstly, the WiFi landmarks are the same as the reference points (RPs) in the typical WiFi fingerprinting system. As the WiFi fingerprinting system operates at the running phase, this module estimates the current position by comparing the current measurement of RSSI with the landmarks. If some conditions are met, the recognition component produces the position of the detected landmark as a calibration position $\left(x_{c}, y_{c}\right)$. If we select the WiFi landmarks carefully, we can get a very good result with a much smaller number of RPs compared with the conventional WiFi fingerprinting systems. The costs of the radio map construction therefore can be reduced. This WiFi landmark detection has two roles. One determines the initial position with the assumption that the user starts from a certain WiFi landmark. The other determines whether the user passes by the area of one WiFi landmark during walking. Next, the turning activities could be used to match a specific location with a given map. The turning landmarks are defined at specific corners (normal turning) and locations of dead ends (U-turning). The module detects the user's behavior through the corners using the inertial sensors. The developed method uses only the changes of heading in consecutive walking steps. Lastly, the detection of the stairs landmark uses the results of the turning detection and floor estimation. In our work, we assumed the shape of the stairs includes an intermediate area, where the user should turn around. This module, therefore, checks the conditions of the changes in altitude and turning behavior.

At every new step detection, the position coordinator determines the current position based on the outputs from both components. The system updates the position with the given position $\left(x_{c}, y_{c}\right)$ of the landmark whenever it was detected.

The two roles of the floor detection component are to estimate the current floor level and to detect the changes between floors. To handle the limitations of using only the barometer, an adaptive approach is suggested. The basic idea is to use the barometric pressure measurements only in short terms. It uses the results of the WiFi module whenever the user does not move. This simple adaptation strategy can help to reduce the search burden for the WiFi landmarks while the user walks, by searching only the landmarks on a given floor. We used the discrete value, $z_{\mathrm{FL}}(t)$, for the floor level.

3.2. Landmark Recognition. This section describes the definition of landmarks and their recognition method in detail. The common data structure of three landmarks is defined as the ID, the type of landmark, the calibration position $\left(x_{c}\right.$, $\left.y_{c}\right)$, the floor level, the data if it is needed\}. The meaning of each attribute could be easily understood by its name. The last field could be any sort of data for the given landmark. For the WiFi landmark, it should be a set of fingerprint data, and it is none for the turning landmark.

3.2.1. WiFi Landmark. As described earlier, the WiFi landmark is the same RP as those in typical WiFi fingerprinting systems, which means that the WiFi landmark has its fingerprint from the training (or radio map building) phase. In our work, the fingerprint is defined as five pieces of paired data, which are the ID of the access point (AP) and the mean value of RSSI from the AP. Five APs with the strongest signal strength when the device collects the signal at the landmark are chosen. The matching error is the Euclidean distance between the measured RSSI and the fingerprint value of the landmark. Our scheme could show good performance for detecting the landmarks even when using a limited number of APs. 


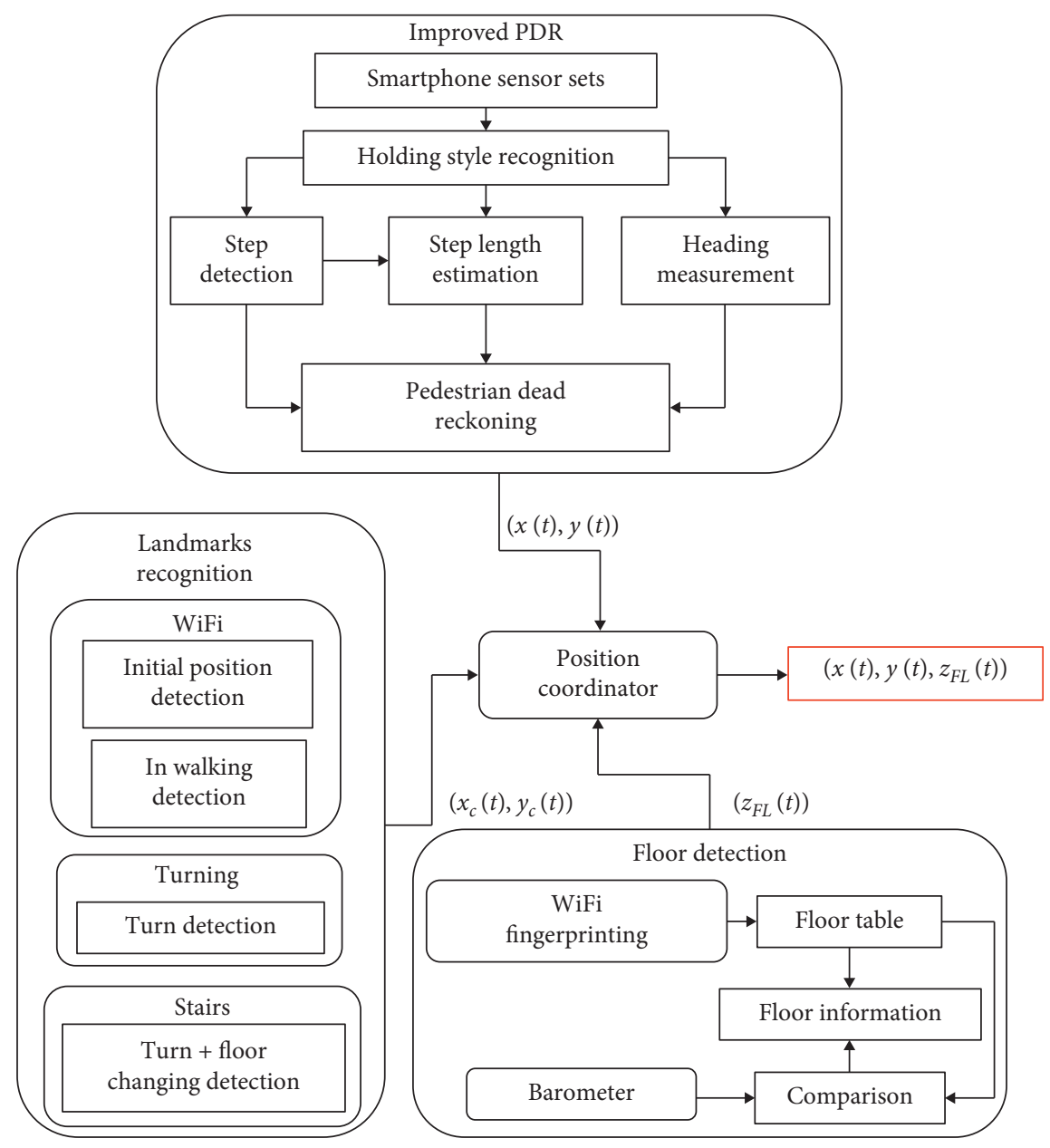

Figure 1: A block diagram of the proposed system.

In the stopping mode, the module estimates the position of the user among all WiFi landmarks as the initial position. Here, we assumed that the initial position of the user is one of the WiFi landmarks. The module finds the landmark that shows the minimum matching error among the landmarks locating on the floor, which is determined from the floor detection component as

$$
(x(t), y(t))=\left(x_{c}, y_{c}\right) \text { of the landmark } \min _{i}\left\{e_{i}(t)\right\},
$$

where $e_{i}(t)$ is the matching error of the landmark $(i)$.

The estimation of the initial position is very important because the PDR can only provide incremental or relative changes in position. Using floor detection, the module could reduce the searching burden.

After the estimation of the initial position, the module operates in the walking mode. When the pedestrian walks, the module finds the events that the user passes through a WiFi landmark. At every scanning of the WiFi signals, the module computes the following features:

F1: find the landmark $(i), \min \left\{e_{i}(t)\right\}$.

F2: calculate the geographic ${ }^{i}$ distance $d_{g}(t)$ as $d_{g}(t)=\left\|(x(t), y(t))-\left(x_{c}, y_{c}\right)\right\|$ of the landmark $(i)$.

F3: calculate the escaped feature, $N_{\text {esc }}(t)$, which is defined as the cumulative number of walking steps since the last WiFi landmark detection event. This is reset to zero when a WiFi calibration occurs. This feature can solve the recalibration problem that occurs before the user escapes the area covered by a WiFi landmark.

Then, the module tests the following conditions:

$$
\begin{aligned}
\min _{i}\left\{e_{i}(t)\right\} & <T h_{1}, \\
d_{g}(t) & <T h_{2}, \\
N_{\mathrm{esc}}(t) & >T h_{3} .
\end{aligned}
$$

If these conditions are met, then the module notifies the coordinator about the detection of a WiFi landmark by providing the calibration position $\left(x_{c}, y_{c}\right)$ of the landmark.

3.2.2. Turning Landmark. The i-PDR component can estimate the heading of walking accurately based on the recognized holding style as shown in our previous work [25]. 
The recognition method for turning landmark uses the change of heading directly. The method uses two features, the average value of the angular velocity, $\omega_{60}$, and the differences between heading changes, $\Delta_{H}$. The calculations involved in these features are given as follows:

$F 1: \omega_{60}(t)$ is calculated as

$$
\omega_{60}(t)=\operatorname{mean}(\|\omega(t-k)\|), \quad k=1, \ldots, 60,
$$

where $\omega$ is the angular velocity vector from the gyroscope sensor and $\omega_{60}$ is the average value of the last 60 samples $(2 \mathrm{~s})$ of the magnitude of the $\omega$ vector.

F2: $\Delta_{H}(t)$ is calculated as

$$
\Delta_{H}(t)=\left|\left(H(t)+H\left(t_{1}\right)\right)-\left(H\left(t_{2}\right)+H\left(t_{3}\right)\right)\right|,
$$

where $H(t)$ is the heading of the current step and $H\left(t_{i}\right)$ is the heading of the $i$-th steps before the current step.

When the i-PDR component detects a new step, the turning module computes two features and tests the following conditions:

$$
\begin{aligned}
& \omega_{60}>T h_{4}, \\
& \Delta_{H}>T h_{5} \longrightarrow \text { Turn. }
\end{aligned}
$$

The feature $\Delta_{H}(t)$ reflects the degree of changes in heading, and the feature $\omega_{60}(t)$ represents the degree of orientation movements.

A pedestrian can randomly turn at places where they are not landmarks, and this can lead to the wrong calibration. To solve this problem, the turning module tests the geographic distance condition (i.e., $\left.d_{g}(t)<T h_{2}\right)$ in a way as same as in the WiFi module. The turning landmark has an inherent disadvantage in that it cannot be defined in an open space without corners.

3.2.3. Stairs Landmark. In this work, we assumed that all stairs have an intermediate area where the user should make turns. Walking in this area can be recognized by detecting the turning behavior and the changes in altitude. Therefore, the stairs module can use the results of the turning detection module. Moreover, it uses the results of the floor detection component. The floor detection component uses the barometer to recognize the walking state such as going up or down on stairs and walking on a plain area. It also estimates the current floor level. When two conditions are met, the system changes the map for the detected floor and updates the user position to calibrate the position based on the detected stairs landmark.

3.3. Floor Detection. A simple but robust floor detection method is suggested. This method wants to take advantage of both the WiFi fingerprinting technique and a barometer sensor. It is easy to recognize the change of altitude of a pedestrian by measuring barometric pressure values using a barometer. The detection of floor changes for a pedestrian, however, requires a technique that is more powerful than the barometer. Specifically, the recognition method needs adaptation capability for the changes of environments and sensors, among others. The proposed method operates in two modes, the stopping mode and the walking mode. In the stopping mode, the component estimates the current floor level using a simple WiFi fingerprinting method. In the walking mode, this component detects the floor change of the pedestrian using a barometer.

In the stopping mode, the component executes a floor recognition method based on a voting strategy. At every scanning of the APs, the scheme calculates the sum of the matching errors at each floor as

$$
S_{e}(i)=\frac{1}{N_{f}^{i}} \sum_{j=1}^{N_{f}^{i}} e_{j}^{i}(t),
$$

where $S_{e}(i)$ is the sum of matching errors at floor $(i), N_{f}^{i}$ is the number of RPs at floor $(i)$, and $e_{j}^{i}(t)$ is the matching error of RP $(j)$ at floor $(i)$. The error is calculated based on the idea from Gansemer et al. [29] as

$$
e_{j}^{i}(t)=\sqrt{\sum_{k=1}^{N^{j}} \frac{\left(\mathrm{RSS}_{k}(t)-\mu_{k}^{j}\right)^{2}}{N^{j}}},
$$

where $\operatorname{RSS}_{k}(t)$ is the measured RSS value of AP $(k), \mu_{k}^{j}$ is the mean value of the AP $(k)$ as a fingerprint of the RP $(j)$, and $N^{j}$ is the number of APs for RP $(j)$.

Then, the scheme chooses the floor that has the minimum value of the sum, $S_{i}$, then adds one point to the floor, and stores the results as a list. The scanning number, $N_{\text {scan }}=30$, is the length of the list. After processing, each floor has its score, and the sum of all scores is $N_{\text {scan. }}$. The floor with the biggest score is the current floor.

In the walking mode, the scheme detects the floor movements by using a barometer. Whenever a new step is detected, the scheme calculates the feature as

$$
\Delta_{B}(t)=b(t)-b\left(t_{1}\right),
$$

where $b(t)$ is the pressure value at the current step and $b\left(t_{1}\right)$ is the pressure value at the previous step. The moving average filter with a window size of 20 samples and an overlap of $60 \%$ is used for the output of the barometer. Then, it tests the following condition:

$$
\left\{\begin{array}{l}
\Delta_{B}(t)<T h_{6} \longrightarrow \mathrm{Up}, \\
\Delta_{B}(t)>T h_{7} \longrightarrow \text { Down, } \\
T h_{7} \leq \Delta_{B}(t) \leq T h_{6} \longrightarrow \text { Plain. }
\end{array}\right.
$$

The recognized current walking state is used to detect the stairs landmark. If the up or down walking is recognized, then the scheme attempts to find the closest floor by comparing the current pressure value against the given floor pressure value from a table called the floor lookup table. The lookup table has a set of pairs such as floor level and the averaged pressure value. The walking state and the current floor level are used to recognize the stairs landmark.

It is known that the device heterogeneity and the weather changes affect greatly the result of barometric pressure measurements. Therefore, the floor level estimation requires 
frequent calibration. Our approach uses the result of the stopping mode. At every stopping mode, the component estimates the current floor by using only the WiFi signal, and then it collects the latest pressure values and updates the lookup table with the calibrated pressure value of the current floor.

3.4. Localization System. The whole proposed localization system is summarized with the pseudocode in Algorithm 1. As input parameters, the proposed system requires the 2dimensional maps of all floors, the database of landmarks, and the initial lookup table of the pressure value-floor pairs. As the first step, the system tries to recognize the current situation of the user among the stopping or walking mode with given landmarks and sensor readings. By using the proposed voting method based on the WiFi RSSI, we can estimate the current floor and the initial position. Then, the system tracks the position of the pedestrian by using the results of the PDR and the landmark recognition components.

\section{Results and Discussion}

4.1. Experimental Setup. To evaluate the performance of the proposed system, several experiments were conducted with three subjects, two males and one female. A three-floor building on our campus was used for evaluation. Four kinds of landmarks are defined in Figure 2 as circles (WiFi), triangles (turning), squares (stairs), and stars (overlapped landmarks of turning and the WiFi). Each landmark has its ID number as (i), where $i=1$ to 23 . The Samsung Galaxy Note 4 was used for the experiments.

We use the thresholds as follows: $T h_{1}=40 \mathrm{~m}, T h_{2}=6.5 \mathrm{~m}$, $T h_{3}=15, T h_{4}=0.5 \mathrm{rad} / \mathrm{sec}, T h_{5}=20$ degrees, $T h_{6}=0.02 \mathrm{hPa}$, and $T h_{7}=-0.02 \mathrm{hPa}$. The whole system is implemented on the smartphone (Android platform), which means the proposed method can run well in real-time.

4.2. Floor and Initial Position Detection. When the subject is not moving, the scheme detects the current floor level and then the initial position among the WiFi landmarks with the same scanned data. It is well known that the WiFi signal is strongly influenced by the orientation of the device, so we have experimented with four major directions at each WiFi landmark. Five trials for each direction were tested on one landmark, so there were 20 trials per landmark. Table 1 shows the results of floor detection, and the average accuracy is $98 \%$ for 300 trials. The floor error occurs mostly when the user is at the landmarks which are nearby the hollow area such as stairs (i.e., landmarks (14), (15), and (19)). In [22], the authors showed that it is not easy to discriminate the differences of the WiFi signals in the hollow area.

Table 2 shows the results of detecting the initial positions. There are five WiFi landmarks on each floor, a total of 15 landmarks on three floors. As mentioned earlier, if floor detection is incorrect, then the initial position cannot be recognized accurately. The cases of landmarks (14), (15), and (19) show the wrong results that were induced from the wrong floor detection. The average error rate of the initial position detection is $2 \%$ over 300 trials. As discussed by Zhao et al. [22], in hollow areas such as stairways, the WiFi signal propagation attenuation between adjacent floors is small. Therefore, our experimental result also shows that the floor detection errors occurred at landmark (15) which is located near the stairways. Table 3 shows the precision, recall, and F-score of the initial position detection results. The worst results are the landmarks (6) of the $3^{\text {rd }}$ floor and (23) of the $1^{\text {st }}$ floor with the precision is 0.91 since sometimes these landmarks are wrongly recognized as the initial position while the pedestrian starts to walk from another initial position such as the landmarks (14) or (15) of the $2^{\text {nd }}$ floor. The recalls of these two landmarks of the $2^{\text {nd }}$ floor are lower than those of others since the system cannot detect these landmarks correctly.

4.3. WiFi, Turning, and Stairs Landmarks Detection. During the walking, the subject holds the smartphone with different holding styles (i.e., HA, CA, SW, and PO). Therefore, we first evaluated the detection performance of three kinds of landmarks while the subjects walk with these four holding styles.

To evaluate the WiFi landmark detection, two subjects walked across $13 \mathrm{WiFi}$ landmarks on three floors (not include landmarks (3) and (21)). For each holding style, the subjects walked six times, which means a total of 78 times crossing the landmarks. Table 4 shows the result. The average error rate is $8.33 \%$. The error rate of the $\mathrm{WiFi}$ landmark detection for the cases of (HA) and (CA) is lower than those of (SW) and (PO). In the (SW) and (PO) cases, the motion of the device is larger than in the other holding styles. We think it is because the big movement has a bigger effect on the RSSI values.

For the turning landmark detection, two subjects walked five times for each holding style on a given path, which includes 14 turning landmarks. Thus, for each holding style, there are 70 times the subjects turned at the corners. As shown in Table 5, the case of (CA) gave the worst result (11.43\%, 8 misses) and the case of (SW) showed the best result (7.14\%, 5 misses). Most errors occurred when changing direction very slowly.

Lastly, two subjects walked a round trip path from the third floor to the first floor. This path includes a total of four times changing the floor. The experiment was repeated 10 times for each holding style, which means there were 40 trials in total for each one. Table 6 shows the results, from the best result (only 2 misses) to the worst result ( 5 misses).

4.4. Tracking Performance. For experiments, three subjects walked on three given paths as shown in Figure 2. The first path is walking on the same floor (the orange line), the second one is traveling over three floors (the green line), and the last one is to go back and forth through the long corridors on the same floor with changing the holding styles during walking (the indigo-blue line). The lengths of the three paths are $48.8 \mathrm{~m}, 277.3 \mathrm{~m}$, and $147.0 \mathrm{~m}$, respectively. 
Input: maps for all floors: $\operatorname{Map}\left(z_{\mathrm{FL}}\right)$

Input: landmark database and the floor lookup table

Input: sensor readings up to current time $t: d_{1: t}$

Output: current floor level and initial position

Output: the detected landmarks

Output: the current position of the pedestrian

(1) CurrentMode $=$ estimateMode $\left(d_{1: t}\right)$

(2) if (CurrentMode $==$ stopping) then

(3) Estimate the floor and then initial position

(4) Update the floor lookup table

(5) end if

(6) else if (CurrentMode == walking) then

(7) Recognize the holding style

(8) if (detect new step) then

(9) if (plain walking) then

(10) Update the position by the PDR

(11) Test for detecting the landmarks (WiFi, turning)

(12) if (detect a landmark) then

(13) Update the current position to landmark position

(14) end if

(15) end if

(16) else if (walking on stairs) then

(17) Test for detecting the stairs landmarks

(18) if (detect a landmark) then

(19) Change the floor level of the current position

(20) Change the floor map

(21) end if

(22) end if

(23) end if

(24) end if

Algorithm 1: Localization method.

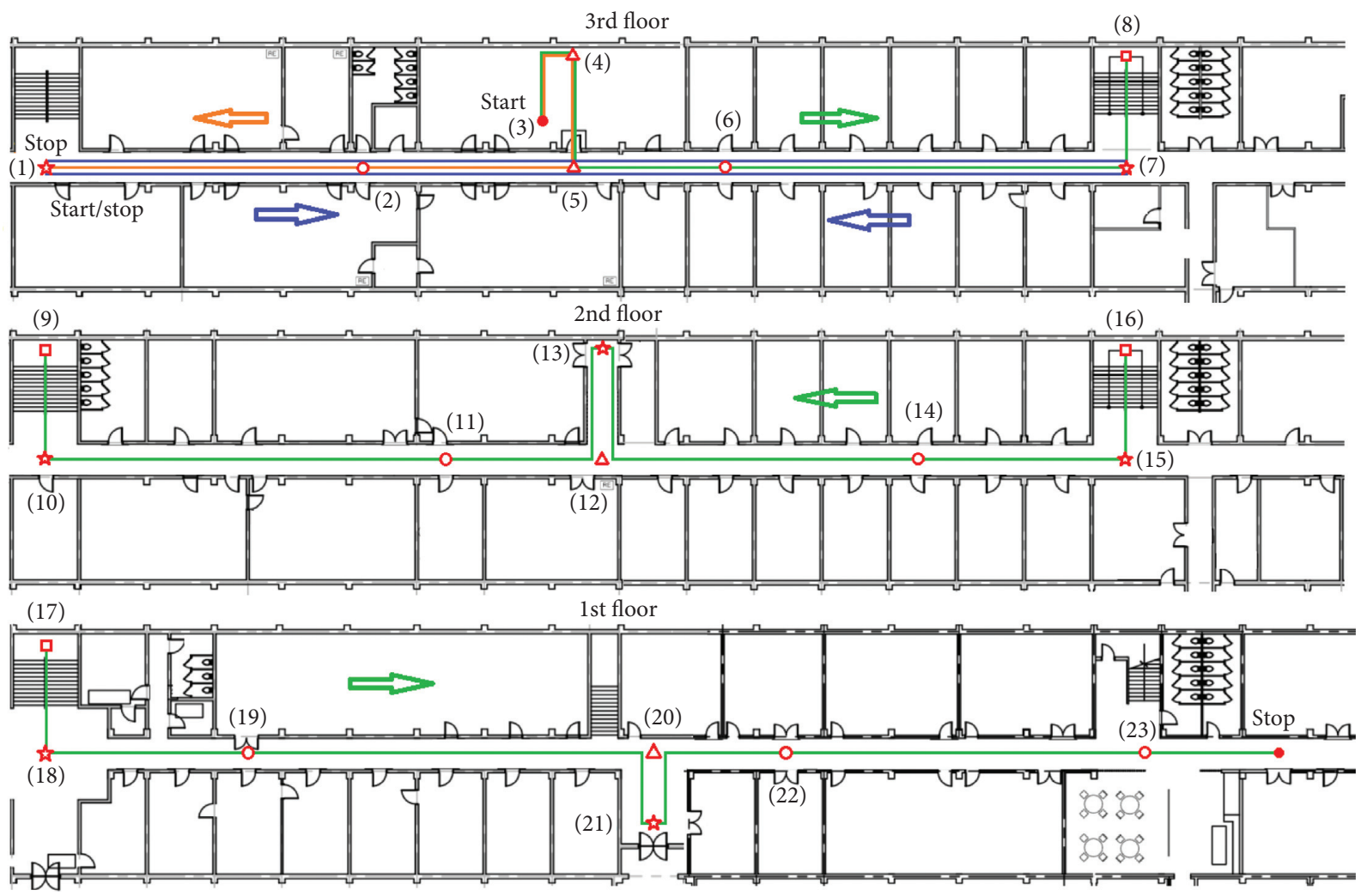

Figure 2: The floor plans, three reference paths, and landmarks. 
TABLE 1: Accuracies and error rates of floor detection.

\begin{tabular}{lcccc}
\hline Recognized & $3^{\text {rd }}$ & $2^{\text {nd }}$ & $1^{\text {st }}$ & Error rate $(\%)$ \\
\hline $3^{\text {rd }}$ & 100 & 0 & 0 & 0.0 \\
$2^{\text {nd }}$ & 3 & 95 & 2 & 5.0 \\
$1^{\text {st }}$ & 0 & 1 & 99 & 1.0 \\
\hline
\end{tabular}

TABLE 2: Errors in initial position detection.

\begin{tabular}{lcccccccc}
\hline Recognized & & $(1)$ & $(2)$ & $(3)$ & $(6)$ & $(7)$ & $\begin{array}{c}\text { Position } \\
\text { error }\end{array}$ & $\begin{array}{c}\text { Floor } \\
\text { error }\end{array}$ \\
\hline \multirow{4}{*}{$3^{\text {rd }}$} & $(1)$ & 20 & 0 & 0 & 0 & 0 & 0 & 0 \\
& $(2)$ & 0 & 20 & 0 & 0 & 0 & 0 & 0 \\
& $(3)$ & 0 & 0 & 20 & 0 & 0 & 0 & 0 \\
& $(6)$ & 0 & 0 & 0 & 20 & 0 & 0 & 0 \\
\multirow{2}{*}{ Recognized } & $(7)$ & 0 & 0 & 0 & 0 & 20 & 0 & 0 \\
\hline \multirow{4}{*}{$2^{\text {nd }}$} & & $(10)$ & $(11)$ & $(13)$ & $(14)$ & $(15)$ & Position & Floor \\
& $(10)$ & 20 & 0 & 0 & 0 & 0 & 0 & 0 \\
& $(11)$ & 0 & 20 & 0 & 0 & 0 & 0 & 0 \\
& $(13)$ & 0 & 0 & 20 & 0 & 0 & 0 & 0 \\
\multirow{2}{*}{ Recognized } & $(14)$ & 0 & 0 & 0 & 17 & 0 & 3 & 3 \\
& $(15)$ & 0 & 0 & 0 & 0 & 18 & 2 & 2 \\
\hline \multirow{4}{*}{$1^{\text {st }}$} & & $(18)$ & $(19)$ & $(21)$ & $(22)$ & $(23)$ & Position & Floor \\
& $(18)$ & 20 & 0 & 0 & 0 & 0 & 0 & 0 \\
& $(19)$ & 0 & 19 & 0 & 0 & 0 & 1 & 1 \\
& $(21)$ & 0 & 0 & 20 & 0 & 0 & 0 & 0 \\
& $(22)$ & 0 & 0 & 0 & 20 & 0 & 0 & 0 \\
& $(23)$ & 0 & 0 & 0 & 0 & 20 & 0 & 0 \\
\hline
\end{tabular}

Table 3: Precisions, recalls, and F-scores of initial position detection.

\begin{tabular}{ccccc}
\hline Floor & Landmark & Precision & Recall & F-score \\
\hline \multirow{4}{*}{$3^{\text {rd }}$} & $(1)$ & 1.00 & 1.00 & 1.00 \\
& $(2)$ & 1.00 & 1.00 & 1.00 \\
& $(3)$ & 0.95 & 1.00 & 0.98 \\
& $(6)$ & 0.91 & 1.00 & 0.95 \\
& $(7)$ & 1.00 & 1.00 & 1.00 \\
\hline \multirow{3}{*}{$2^{\text {nd }}$} & $(10)$ & 1.00 & 1.00 & 1.00 \\
& $(11)$ & 0.95 & 1.00 & 0.98 \\
& $(13)$ & 1.00 & 1.00 & 1.00 \\
& $(14)$ & 1.00 & 0.85 & 0.92 \\
& $(15)$ & 1.00 & 0.90 & 0.95 \\
\hline \multirow{3}{*}{$1^{\text {st }}$} & $(18)$ & 1.00 & 1.00 & 1.00 \\
& $(19)$ & 1.00 & 0.95 & 0.97 \\
& $(21)$ & 1.00 & 1.00 & 1.00 \\
& $(22)$ & 1.00 & 1.00 & 1.00 \\
& $(23)$ & 0.91 & 1.00 & 0.95 \\
\hline
\end{tabular}

We assumed that the subjects walk at a normal speed. Each subject walked three times for a path with a holding style.

Figure 3 shows the trajectories of the proposed method and the i-PDR method for walking on the first path with four holding styles. In Figure 3, the black line is the reference path, while the dots and the stars represent the estimated positions of both methods. The four colors represent four holding styles, respectively. The figure shows that most of the
TABLE 4: Errors in WiFi landmark detection.

\begin{tabular}{lcccc}
\hline & HA & CA & SW & PO \\
\hline Missed & 3 & 4 & 10 & 9 \\
Error (\%) & 3.85 & 5.13 & 12.82 & 11.54 \\
\hline
\end{tabular}

TABLE 5: Errors in turning landmark detection.

\begin{tabular}{lcccc}
\hline & HA & CA & SW & PO \\
\hline Missed & 7 & 8 & 5 & 6 \\
Error (\%) & 10 & 11.43 & 7.14 & 8.57 \\
\hline
\end{tabular}

TABLE 6: Errors in stairs landmark detection.

\begin{tabular}{lcccc}
\hline & HA & CA & SW & PO \\
\hline Missed & 2 & 4 & 5 & 4 \\
Error (\%) & 5 & 10 & 12.5 & 10 \\
\hline
\end{tabular}

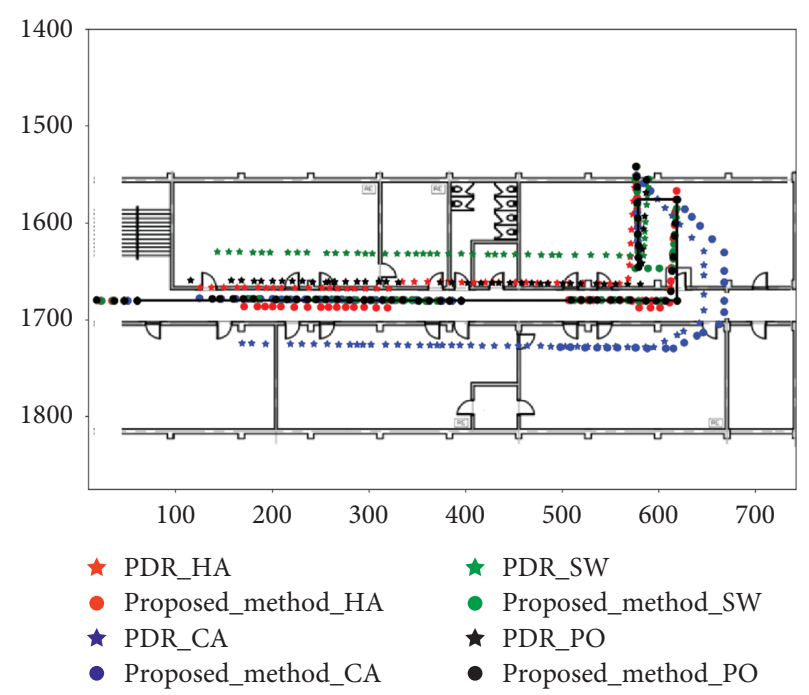

Figure 3: Trajectories of two methods for four holding styles on the first path.

estimated positions of the proposed method are on the reference path except for the first part of the (CA) case by detection of the four landmarks $(1,2,4,5)$. As summarized in Table 7, the errors at the final position show that the proposed method is superior to the i-PDR method in all cases of four holding styles. The average values of both measures (mean and RMSE) for four holding styles show that the proposed method is much less than the i-PDR. Figure 4 shows the box plots of these errors. An interesting thing is that the interquartile range of the proposed method is very small compared with the i-PDR. We think that the correction by detecting the landmarks during walking produces this result. All four holding styles showed a reduction of errors, but the (PO) and (SW) cases especially decreased dramatically.

To calculate the position error of each walking step, we need to know the true position of the step. In this work, the 
TABLE 7: Mean error and RMSE for 4 holding styles at the final position.

\begin{tabular}{lcccccccr}
\hline $\begin{array}{l}\text { Holding style } \\
\text { Method }\end{array}$ & i-PDR & Proposed & i-PDR & Proposed & i-PDR & Proposed & i-PDR & Proposed \\
\hline Mean error $(\mathrm{m})$ & 5.74 & 2.59 & 5.33 & 1.77 & 3.63 & 2.42 & 3.48 \\
RMSE (m) & 6.06 & 2.69 & 5.83 & 2.07 & 4.17 & 2.55 & 3.93 & 2.64 \\
\hline
\end{tabular}

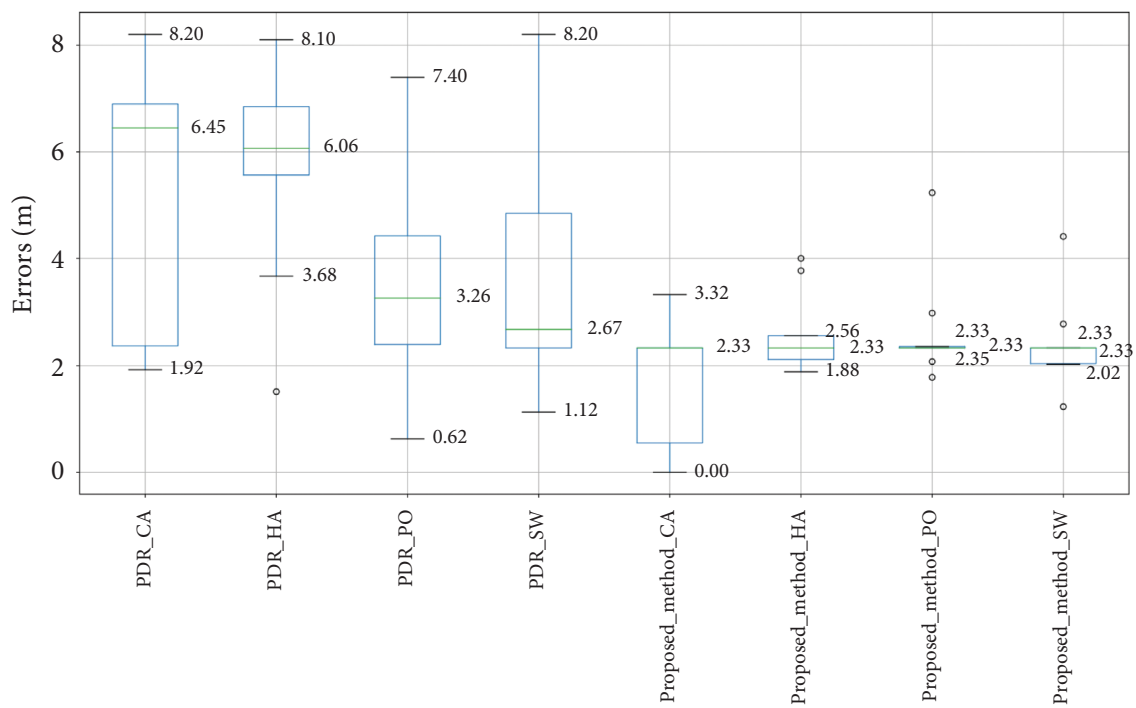

Figure 4: Box plots of the errors for the first path experiment.

virtual points were created on the reference path using a fixed-sized step length, and the position error was calculated by one-to-one mapping of the estimated point and the virtual one in order. Of course, this simple method has a problem in that the error increases in proportion to the difference in the number of the detected steps. However, since the subject's stride length would be quite constant, the error made by this method does not have a significant effect on the comparative analysis. Figure 5 shows the cumulative position error distributions in this experiment. Note that the proposed method showed a big difference in performance improvement according to the holding styles. In the case of (CA) and (SW), there are few differences in performance between the two methods. For this reason, as shown in Figure 5(b), the proposed method for all holding styles shows smaller errors under the $90^{\text {th }}$ percentile but shows almost the same error afterward. Statistically, the occasional recalibration can reduce the error of many estimated positions, but it does not have much effect on a small number of extremes that make a large error.

Figure 6 shows the trajectories of the proposed and the i-PDR methods when a subject walked with a holding style (HA) on the second path. The second path is a one-way route including floor change from third to first as shown in three plans in Figure 6. The blue dots represent the trajectory of the proposed method, and the orange stars represent the result of the i-PDR method. The red stars represent that the system detects one landmark and then calibrates the current position to the given position of the landmark. The green pentagons show the positions of one step before being calibrated. As shown in the figure, the short-range jumps from the green pentagons to the red stars are the effect of the calibration upon detection of the landmarks. For comparison, we set the starting points of the i-PDR method on each floor to be the same as the proposed method because the i-PDR can produce only two-dimensional positions. Both methods show acceptable results for a straight path, driven using the simple quantized method for measuring the heading. The i-PDR method, however, shows a relatively large positional error.

As an example, Figure 7 shows the errors of the proposed and the i-PDR methods at the beginning of this experiment (part as shown in the red rectangle in Figure 6). The green circles highlight the events of landmark detection, specifically three landmarks $(4,6,7)$ on the third floor.

Table 8 shows basic statistics of the calibrated distances (i.e., the size of short-range jumps) by detecting the landmarks for the second path experiment. In this path, the subjects walked through a sequence of 20 landmarks. The sequence is $(4,5,6,7,8)$ on the third floor, eight landmarks $(15,14,12,13,12,11,10,9)$ on the second floor, and seven landmarks $(18,19,20,21,20,22,23)$ on the first floor in order. As shown in Table 8 , the system shows different numbers of landmark detection for each holding style, and many detection errors occurred in the sequences $(12,13,12)$ and $(20,21,20)$. Despite the low detection rate of $68.75 \%$ 


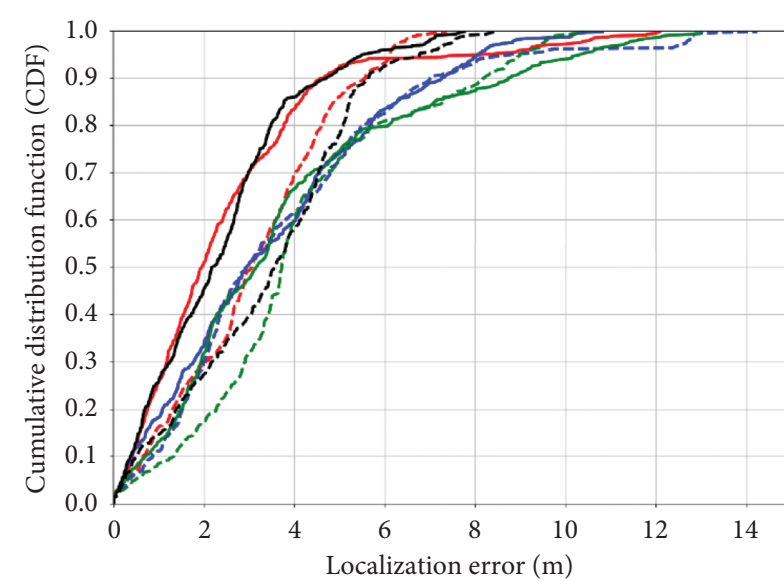

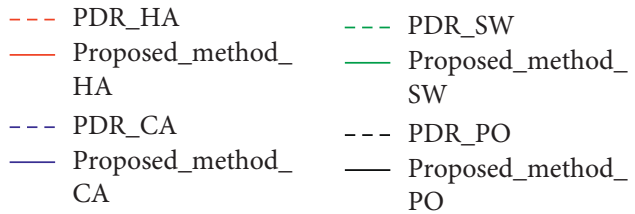

(a)

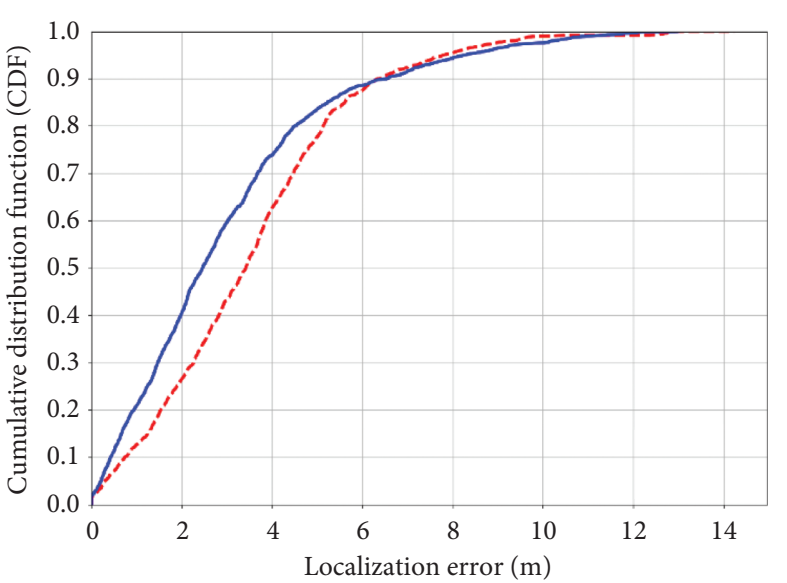

-. - PDR

_ Proposed_method

(b)

FIgURe 5: Cumulative position error distributions for the first path experiment: (a) by four holding styles and (b) without considering holding style.
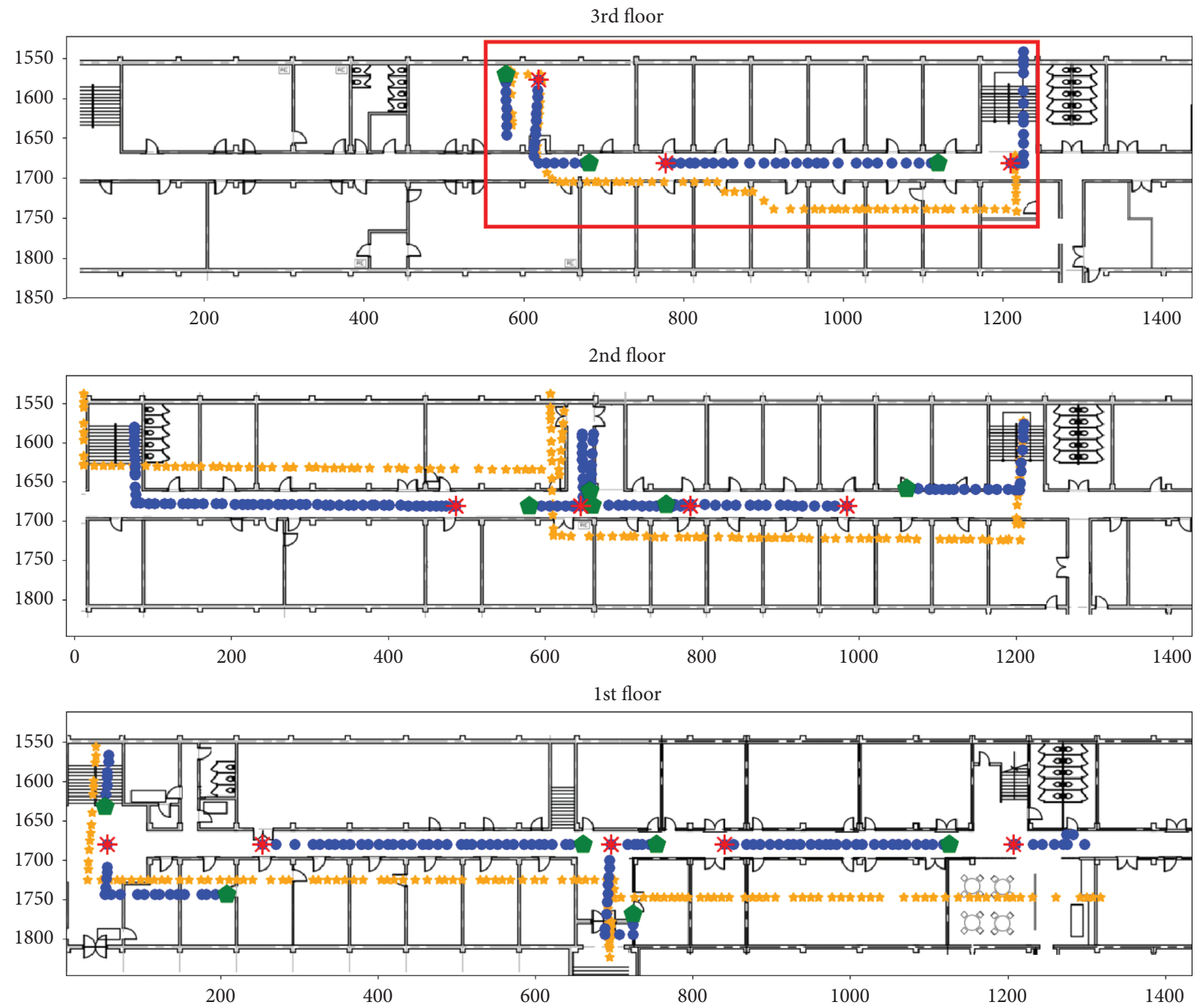

Figure 6: Trajectories of two methods for a holding style (HA) on the second path. 


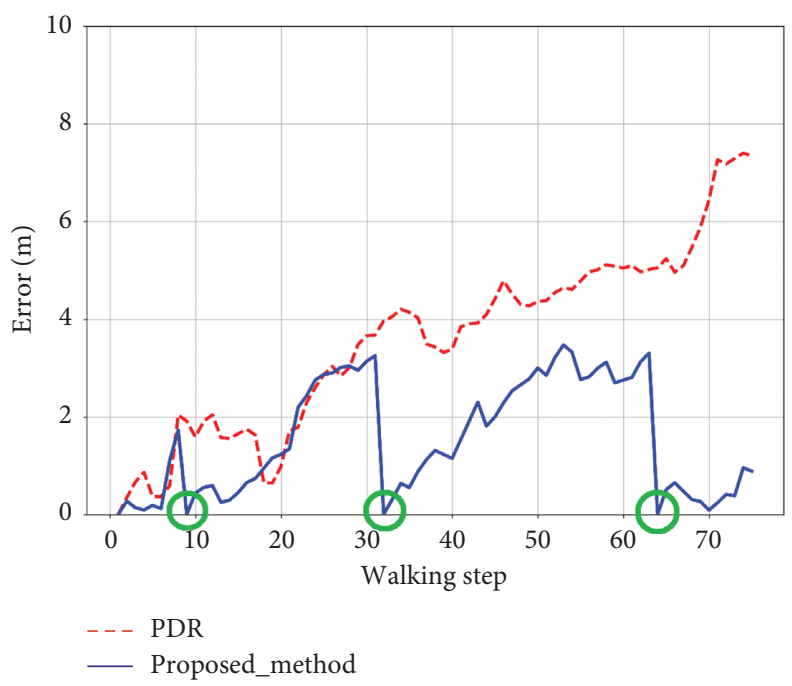

Figure 7: Position errors of two methods at the beginning part.

TABLE 8: Statistics of calibration distances by detection of landmarks.

\begin{tabular}{|c|c|c|c|c|c|}
\hline Holding style & HA & $\mathrm{CA}$ & SW & $\mathrm{PO}$ & Avg. \\
\hline No. of detected landmarks & 13 & 13 & 14 & 15 & 13.75 \\
\hline Detection ratio (\%) & 65.0 & 65.0 & 70.0 & 75.0 & 68.75 \\
\hline Max distance $(\mathrm{m})$ & 4.11 & 5.19 & 5.81 & 4.64 & 4.94 \\
\hline Min distance $(\mathrm{m})$ & 1.75 & 1.65 & 1.03 & 0.66 & 1.27 \\
\hline Mean distance $(\mathrm{m})$ & 3.37 & 3.34 & 3.27 & 2.27 & 3.06 \\
\hline Standard deviation $(\mathrm{m})$ & 0.95 & 1.30 & 1.71 & 1.37 & 1.33 \\
\hline
\end{tabular}

averagely, the proposed method showed accurate tracking performance compared with the i-PDR method through an average calibration distance of $3.06 \mathrm{~m}$.

Figure 8 shows the cumulative position error distributions in the second path experiment. At the $50^{\text {th }}$ percentile, the errors of the proposed method for holding styles (HA), (CA), (SW), and (PO) are $2.83 \mathrm{~m}, 3.04 \mathrm{~m}, 3.22 \mathrm{~m}$, and $3.09 \mathrm{~m}$, and at the $70^{\text {th }}$ percentile, the errors are $4.16 \mathrm{~m}, 4.07 \mathrm{~m}$, $4.88 \mathrm{~m}$, and $5.39 \mathrm{~m}$, respectively. These are quite smaller than the results of the i-PDR method at the same percentiles $0.62 \mathrm{~m}$ to $1.46 \mathrm{~m}$ with an average is $0.91 \mathrm{~m}$. The average RMSE values at the final point for four holding styles are $6.80 \mathrm{~m}$ for the i-PDR method and $3.51 \mathrm{~m}$ for the proposed method. This result shows that the proposed method has $39.57 \%$ to $57.51 \%$ less error compared with the i-PDR method for different holding styles.

In the third path, the subjects started to walk from the landmark (1) and through (2) and (6), made a turn at (7), and come back to (1) to finish a round trip. The subjects walked from the landmark (1) to (6) with the holding style (HA), then changed to (CA) at the landmark (6), changed to (SW) at (7), and changed to (PO) at (6) again until (1). The change of holding style was made without stopping walking. The total number of trials is 9 . Figure 9 shows the trajectories of the proposed method and the i-PDR method for one trial. The dots and the stars represent the proposed method and the i-PDR method, respectively, and the colors represent the four holding styles. The black line is the reference path. As can be seen in Figure 9, most of the results of the proposed method are on the reference path except the segment from (7) to (6) with the holding style (SW). Analysis at the final point also shows the superiority of the proposed method such that the average RMSE values are $2.70 \mathrm{~m}$ for the proposed method and $3.68 \mathrm{~m}$ for the i-PDR method. Figure 10 shows the distributions of the errors of the two methods at the final point by box plots. The figure shows that the proposed method can reduce both the error and the variance compared with the i-PDR method. Lastly, the cumulative error distributions of the two methods are shown in Figure 11 by using the same method to make virtual points. As can be seen in Figure 11, the proposed method could reduce the error size of all data for simple and shortlength paths such as the third path.

It is not easy to directly and fairly compare the performance of our proposed method to other works, but we attempted to compare them in some respects. All of the selected studies are based on an approach that combines the PDR technique with corrections from different methods, which is the same idea as our proposed scheme. The comparison is shown in Table 9. We compared the proposed method with six evaluation factors to show the features of the proposed method. The results of other studies were analyzed based on their literature. The fusion methods in column "Technique" are divided into two categories: the triggering method and various filtering methods. The triggering methods refer to update to a given specific position 


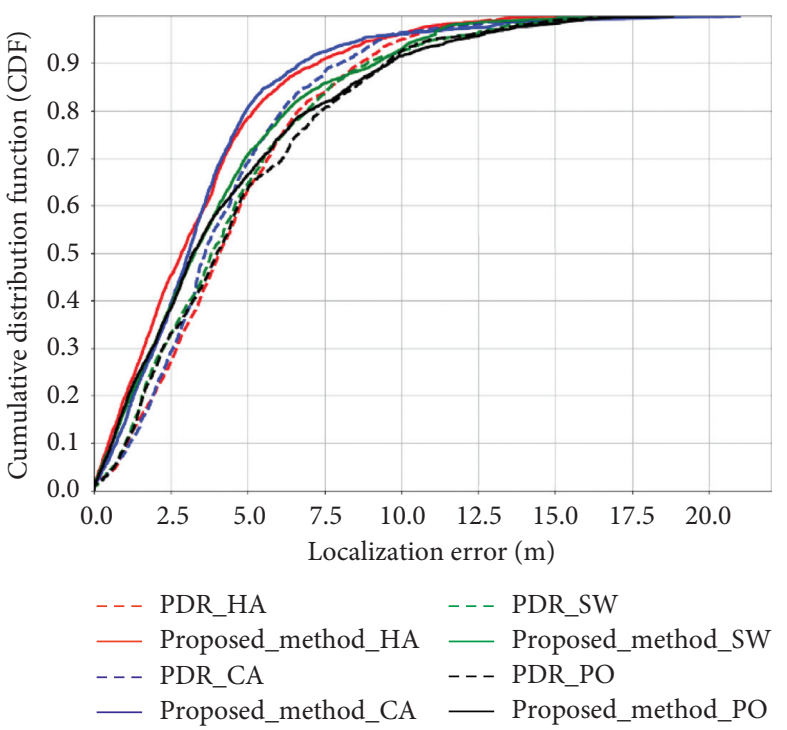

(a)

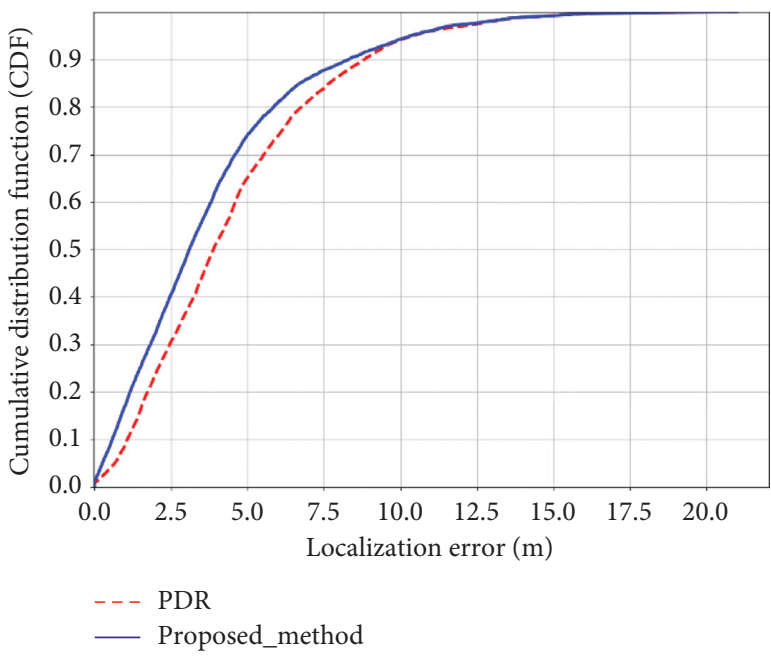

(b)

Figure 8: Cumulative position error distributions for the second path experiment: (a) by four holding styles and (b) without considering holding style.

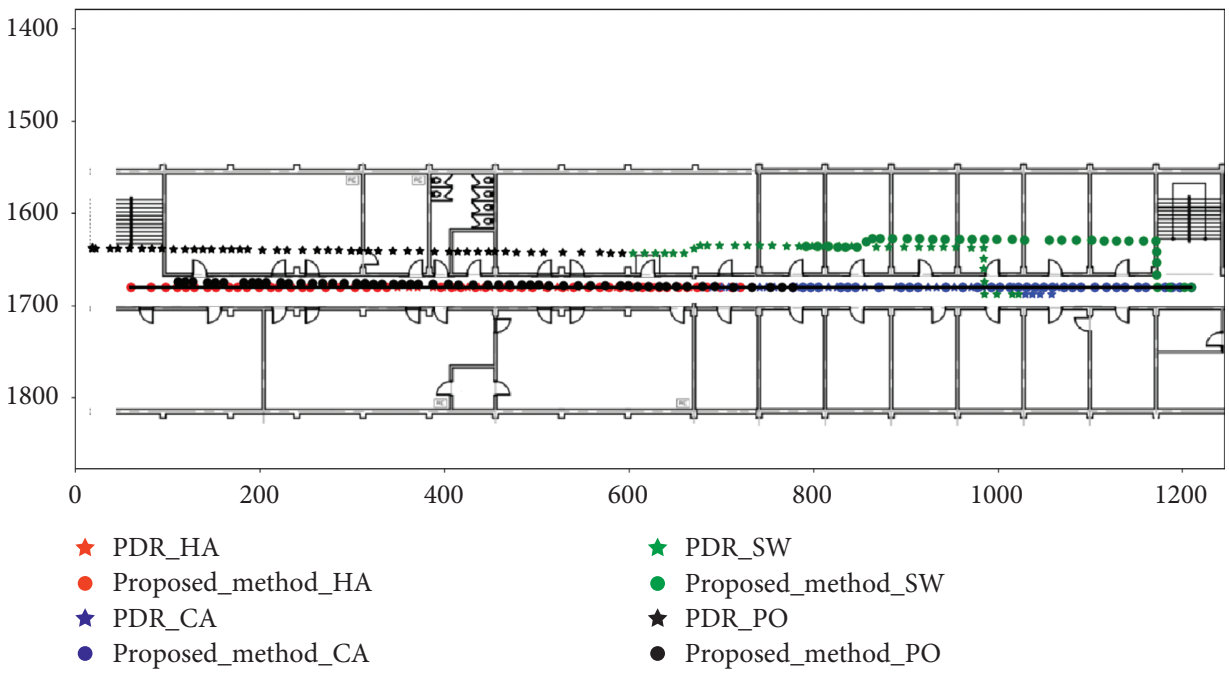

Figure 9: Trajectories of two methods for the third path experiment.

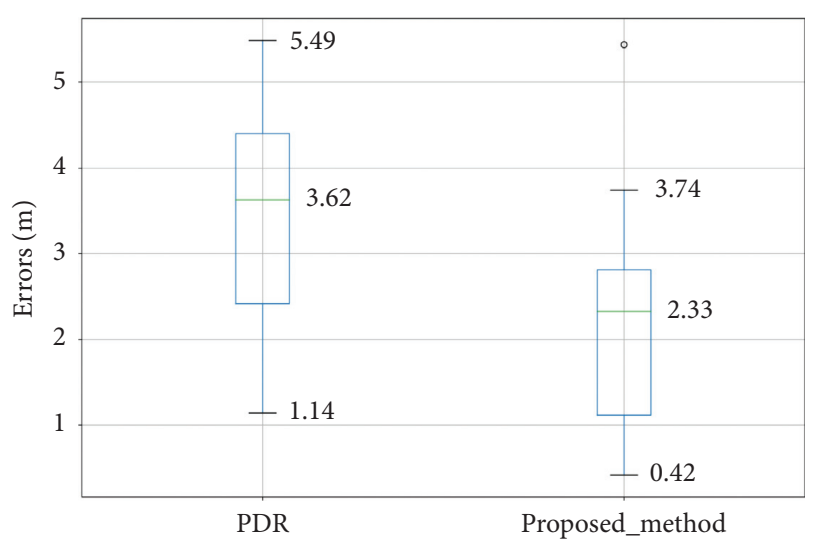

Figure 10: Box plots of the errors for the third path experiment. when a system recognizes the position of a pedestrian using other information. The other studies were developed by applying the EKF, UKF, and PF methods. Note that as the table shows, recent studies $[13,31]$, including the proposed method, provide a method to handle various holding styles. The system in [12] achieved a positioning accuracy of $1.5 \mathrm{~m}$; however, their experimental setup is simple with a straight line and an L-shape path. In $[14,30]$, their proposed methods are not running in real-time since they collected data from sensors and analyzed the results on the offline tools. Moreover, they tested their system in only one scenario and with only one holding style. The works in $([9,11,13,31]$ reported good results with the accuracy that can reach $1 \mathrm{~m}$ using the complex filters. As a matter of course, complex filters require a lot of computational costs and power 


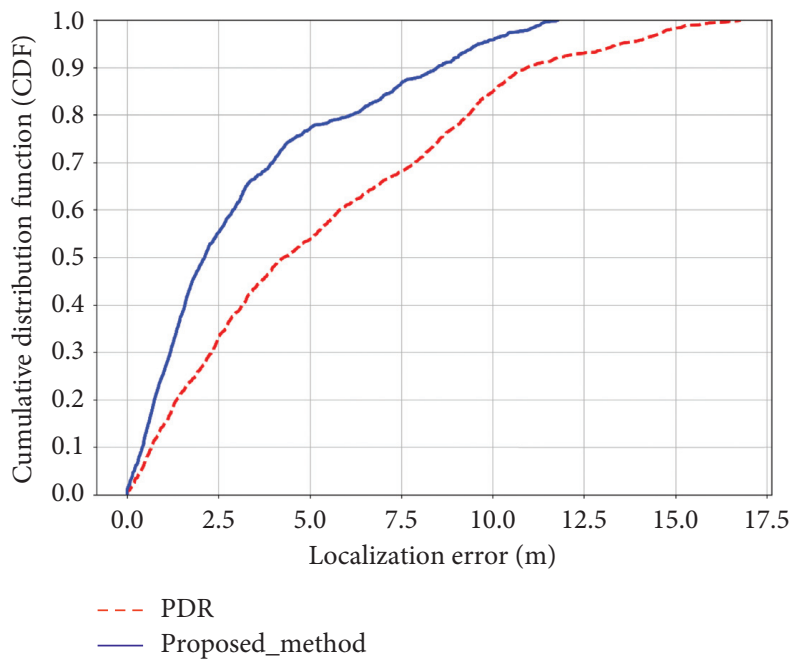

Figure 11: Cumulative position error distributions for the third path experiment.

TABLE 9: Comparison of the proposed method and existing studies.

\begin{tabular}{|c|c|c|c|c|c|c|}
\hline Ref. no. (year) & Technique (fusion method) & Real-time & Holding styles & Test scenarios & Positioning accuracy & 3D capability \\
\hline Proposed method & PDR + LM (triggering) & Yes & 4 & 3 & $<3.1 \mathrm{~m}$ & Yes \\
\hline$[14](2016)$ & PDR + LM (triggering) & No & 1 & 1 & $2.17 \mathrm{~m}$ & No \\
\hline [30] (2016) & $\mathrm{PDR}+\mathrm{MM}$ (triggering) & No & 1 & 1 & $2.5 \mathrm{~m}$ & Yes \\
\hline [12] (2018) & $\mathrm{PDR}+\mathrm{WiFi}$ (triggering) & Yes & $\mathrm{n} / \mathrm{a}$ & 2 & $1.5 \mathrm{~m}$ & No \\
\hline [11] (2016) & $\mathrm{PDR}+\mathrm{WiFi}+\mathrm{LM}(\mathrm{EKF})$ & No & 1 & 2 & $1.22 \mathrm{~m}$ & No \\
\hline [9] (2019) & $\mathrm{PDR}+\mathrm{MM}(\mathrm{PF})$ & Yes & 1 & 3 & $4.45 \mathrm{~m}$ & Yes \\
\hline [13] (2019) & $\mathrm{PDR}+\mathrm{WiFi}(\mathrm{UKF})$ & No & 4 & 1 & $1.46 \mathrm{~m}$ & No \\
\hline [31] (2020) & $\mathrm{PDR}+\mathrm{MM}(\mathrm{PF})$ & Yes & 5 & 2 & $2.2 \mathrm{~m}$ & No \\
\hline
\end{tabular}

EKF: extended Kalman filter; LM: landmark; MM: map matching; PF: particle filter; UKF: unscented Kalman filter.

consumption. Some of them $[9,30]$ can track the position of the user in multi-floor buildings. The work in [9] reported the $4.45 \mathrm{~m}$ errors in a multi-floor path of $363.22 \mathrm{~m}$ length; meanwhile, our proposed system gets an error of $3.51 \mathrm{~m}$ for a multi-floor path of $277.3 \mathrm{~m}$.

\section{Conclusions}

This paper presented a pedestrian localization system that uses an improved PDR technique with the recognition of four holding styles and a map matching technique by detecting different landmarks. In this work, three kinds of landmarks, the WiFi, the turning, and the stairs, are defined, and the detection methods are proposed. Based on the detection of various landmarks, the system could reduce drastically the cumulative errors from the PDR method. Moreover, a simple but robust floor detection method is proposed for estimating the initial position and changes between floors. These capabilities can be extended from the $2 \mathrm{D}$ positioning to a $3 \mathrm{D}$ space (e.g., a multi-floor building). The system can reduce the error up to $57.51 \%$, showing results that are more accurate than the results based on the improved PDR alone for different holding styles. In conclusion, simply calibrating the position in the conventional PDR system, especially SHSs, using different kinds of location-related information (defined as landmarks) that can be found in the environment can significantly improve the performance of indoor positioning systems. Therefore, it is important to find a landmark that is effective in improving the performance of the PDR system.

However, the proposed method has some limitations. First, if the user walks through large open spaces such as huge shopping malls or airports rather than regular office buildings, the turning landmarks are not useful because there are not many corners structurally. This landmark is more suitable for buildings with many corners. The second limitation is that the tracking performance of the proposed method is proportional to the density of the landmark and its detecting performance. In other words, the dense landmarks and high detection capabilities can make frequent error corrections, which greatly improves the performance of the system. However, if the landmark density is low in environments and/or cannot be detected well, the performance is almost the same as the PDR only. Therefore, in order to obtain a certain level of location performance, it must be required that a certain level of landmark density and detection performance. Besides, from the Android 9 version, the scanning interval for the $\mathrm{WiFi}$ module increases to 30 seconds per scan. This means that we can no longer use the current WiFi landmark.

In the future, we are trying to find more diverse landmarks that can be used in various environments and develop 
methods to detect them. We will also research the effective fusion methods combining the PDR technique and the landmark detection method.

\section{Data Availability}

The data used to support the findings of this study are available from the corresponding author upon request.

\section{Conflicts of Interest}

The authors declare that they have no conflicts of interest.

\section{Acknowledgments}

This work was supported by the Hallym University Research Fund, 2018 (HRF-201802-013).

\section{References}

[1] R. Harle, "A survey of indoor inertial positioning systems for pedestrians," IEEE Communications Surveys \& Tutorials, vol. 15, no. 3, pp. 1281-1293, 2013.

[2] G. Chen, X. Meng, Y. Wang, Y. Zhang, P. Tian, and H. Yang, "Integrated WiFi/PDR/Smartphone using an Unscented Kalman filter algorithm for 3D indoor localization," Sensors, vol. 15, no. 9, pp. 24595-24614, 2015.

[3] Y. Zhuang, H. Lan, Y. Li, and N. El-Sheimy, "PDR/INS/WiFi integration based on handheld devices for indoor pedestrian navigation," Micromachines, vol. 6, no. 6, pp. 793-812, 2015.

[4] Y. Zhuang, J. Yang, Y. Li, L. Qi, and N. El-Sheimy, "Smartphone-based indoor localization with Bluetooth low energy beacons," Sensors, vol. 16, no. 5, pp. 596-616, 2016.

[5] Y. Li, Y. Zhuang, P. Zhang, H. Lan, X. Niu, and N. El-Sheimy, "An improved inertial/WiFi/magnetic fusion structure for indoor navigation," Information Fusion, vol. 34, pp. 101-119, 2017.

[6] C. Ascher, C. Kessler, M. Wankerl, and G. F. Trommer, "Dual IMU indoor navigation with Particle filter based mapmatching on a smartphone," in Proceedings of the 2010 International Conference on Indoor Positioning and Indoor Navigation, pp. 1-5, Zurich, Switzerland, September 2010.

[7] H. Xie, T. Gu, X. Tao, H. Ye, and J. Lu, "A reliability-augmented Particle filter for magnetic fingerprinting based indoor localization on smartphone," IEEE Transactions on Mobile Computing, vol. 15, no. 8, pp. 1877-1892, 2016.

[8] S. Knauth, "Smartphone PDR positioning in large environments employing WiFi, particle filter, and backward optimization," in Proceedings of the 2017 International Conference on Indoor Positioning and Indoor Navigation, pp. 1-6, Sapporo, Japan, 2017.

[9] H. Zhao, W. Cheng, N. Yang, S. Qiu, Z. Wang, and J. Wang, "Smartphone-based 3D indoor pedestrian positioning through multi-modal data fusion," Sensors, vol. 19, no. 20, pp. 4554-4574, 2019.

[10] Z. Chen, H. Zou, H. Jiang, Q. Zhu, Y. Soh, and L. Xie, "Fusion of WiFi, smartphone sensors and landmarks using the Kalman filter for indoor localization," Sensors, vol. 15, no. 1, pp. 715-732, 2015.

[11] Z.-A. Deng, G. Wang, D. Qin, Z. Na, Y. Cui, and J. Chen, "Continuous indoor positioning fusing WiFi, smartphone sensors and landmarks," Sensors, vol. 16, no. 9, pp. 1427-1447, 2016.
[12] L.-F. Shi, Y. Wang, G.-X. Liu, S. Chen, Y.-L. Zhao, and Y.-F. Shi, "A fusion algorithm of indoor positioning based on PDR and RSS fingerprint," IEEE Sensors Journal, vol. 18, no. 23, pp. 9691-9698, 2018.

[13] J. Yu, Z. Na, X. Liu, and Z. Deng, "WiFi/PDR integrated indoor localization using unconstrained smartphones," EURASIP Journal on Wireless Communications and Networking, vol. 2019, no. 1, pp. 41-54, 2019.

[14] X. Wang, M. Jiang, Z. Guo, N. Hu, Z. Sun, and J. Liu, “An indoor positioning method for smartphones using landmarks and PDR," Sensors, vol. 16, no. 12, pp. 2135-2152, 2016.

[15] B. Zhou, Q. Li, Q. Mao, W. Tu, and X. Zhang, "Activity sequence-based indoor pedestrian localization using smartphones," IEEE Transactions on Human-Machine Systems, vol. 45, no. 5, pp. 562-574, 2015.

[16] M. Elhoushi, J. Georgy, A. Noureldin, and M. J. Korenberg, "Motion mode recognition for indoor pedestrian navigation using portable devices," IEEE Transactions on Instrumentation and Measurement, vol. 65, no. 1, pp. 208-221, 2016.

[17] Y. Shu, C. Bo, G. Shen, C. Zhao, L. Li, and F. Zhao, "Magicol: indoor localization using pervasive magnetic field and opportunistic WiFi sensing," IEEE Journal on Selected Areas in Communications, vol. 33, no. 7, pp. 1443-1457, 2015.

[18] G. Wang, X. Wang, J. Nie, and L. Lin, "Magnetic-based indoor localization using smartphone via a fusion algorithm," IEEE Sensors Journal, vol. 19, no. 15, pp. 6477-6485, 2019.

[19] X. Li, J. Wang, and C. Liu, "A Bluetooth/PDR integration algorithm for an indoor positioning system," Sensors, vol. 15, no. 10, pp. 24862-24885, 2015.

[20] F. Seco and A. R. Jiménez, "Smartphone-based cooperative indoor localization with RFID technology," Sensors, vol. 18, no. 1, pp. 266-289, 2018.

[21] K. Park, H. Shin, and H. Cha, "Smartphone-based pedestrian tracking in indoor corridor environments," Personal and Ubiquitous Computing, vol. 17, no. 2, pp. 359-370, 2013.

[22] F. Zhao, H. Luo, X. Zhao, Z. Pang, and H. Park, "HYFI: hybrid floor identification based on wireless fingerprinting and barometric pressure," IEEE Transactions on Industrial Informatics, vol. 13, no. 1, pp. 330-341, 2017.

[23] R. Elbakly, H. Aly, and M. Youssef, "TrueStory: accurate and robust RF-based floor estimation for challenging indoor environments," IEEE Sensors Journal, vol. 18, no. 24, pp. 10115-10124, 2018.

[24] X. Shen, Y. Chen, J. Zhang, L. Wang, G. Dai, and T. He, "BarFi: barometer-aided Wi-Fi floor localization using crowdsourcing," in Proceedings of the 2015 IEEE 12th International Conference on Mobile Ad Hoc and Sensor Systems, pp. 416-424, Dallas, TX, USA, 2015.

[25] K. Nguyen-Huu, K. H. Lee, C. G. Song, and S.-W. Lee, "A heading estimation based on smartphone holding styles," in Proceeding of the HCI Korea, pp. 1-7, Jeongseon, Republic of Korea, 2016.

[26] K. Nguyen-Huu, C. G. Song and S.-W. Lee, "Smartphone holding styles based step detection and length estimation," Journal of Information Science and Engineering, vol. 35, no. 3, pp. $537-554,2019$.

[27] C. Combettes and V. Renaudin, "Walking direction estimation based on statistical modeling of human gait features with handheld MIMU," IEEE/ASME Transactions on Mechatronics, vol. 22, no. 6, pp. 2502-2511, 2017.

[28] H. Ju, S. Y. Park, and C. G. Park, "A smartphone-based pedestrian dead reckoning system with multiple virtual tracking for indoor navigation," IEEE Sensors Journal, vol. 18, no. 16, pp. 6756-6764, 2018. 
[29] S. Gansemer, U. Großmann, and S. Hakobyan, "RSSI-based Euclidean distance algorithm for indoor positioning adapted for the use in dynamically changing WLAN environments and multi-level buildings," in Proceedings of the 2010 International Conference on Indoor Positioning and Indoor Navigation, pp. 1-6, Zurich, Switzerland, 2010.

[30] F. Gu, A. Kealy, K. Khoshelham, and J. Shang, "Efficient and accurate indoor localization using landmark graphs," ISPRSInternational Archives of the Photogrammetry, Remote Sensing and Spatial Information Sciences, vol. XLI-B2, pp. 509-514, 2016.

[31] X. Wang, G. Chen, M. Yang, and S. Jin, "A multi-mode PDR perception and positioning system assisted by map matching and particle filtering," ISPRS International Journal of GeoInformation, vol. 9, no. 2, pp. 93-116, 2020. 